\section{Cahiers de Narratologie}

Analyse et théorie narratives

13 | 2006

Nouvelles approches de l'intertextualité

\title{
La répétition interrompue ou peindre l'Histoire, un polylogue intertextuel
}

Jacques La Mothe

\section{(2) OpenEdition}

Journals

Édition électronique

URL : http://journals.openedition.org/narratologie/338

DOI : $10.4000 /$ narratologie.338

ISSN : 1765-307X

Éditeur

LIRCES

Référence électronique

Jacques La Mothe, «La répétition interrompue ou peindre l'Histoire, un polylogue intertextuel», Cahiers de Narratologie [En ligne], 13 | 2006, mis en ligne le 01 septembre 2006, consulté le 20 avril 2019. URL : http://journals.openedition.org/narratologie/338 ; DOI : 10.4000/narratologie.338

Ce document a été généré automatiquement le 20 avril 2019.

\section{(c) $($ i) $(9)$}

Cahiers de Narratologie - Analyse et théorie narratives est mis à disposition selon les termes de la licence Creative Commons Attribution - Pas d'Utilisation Commerciale - Pas de Modification 4.0 International. 


\title{
La répétition interrompue ou peindre l'Histoire, un polylogue intertextuel
}

\author{
Jacques La Mothe
}

1 Alors qu'il vient de terminer la Bataille de Taillebourg, Delacroix, en 1838, reçoit une seconde commande pour le musée historique de Versailles, L'entrée des Croisés à Constantinople, 1204, destinée à la salle des Croisades. La toile est exposée au Salon de 1841 ; le livret en donne le commentaire suivant :

« Baudouin, comte de Flandre, commandait les Français qui avaient donné l'assaut du côté de la terre, et le vieux doge Dandolo, à la tête des Vénitiens, et sur ses vais seaux, avait attaqué le port. / Les principaux chefs parcourent les divers quartiers de la ville, et les familles éplorées viennent sur leur passage invoquer leur clémence ».

2 La Quatrième Croisade, ordonnée par Innocent III pour libérer Jérusalem tenue par les Musulmans depuis 1187, n'atteignit jamais son but. Les croisés, au lieu de combattre les infidèles, s'étaient mis d'abord au service des Vénitiens, qui leur fournissaient une flotte, puis, intervenant dans les affaires des Grecs, s'étaient emparés à deux reprises de Constantinople établissant leur domination sur des populations schismatiques du point de vue romain, mais néanmoins chrétiennes. La ville fut mise à sac, et Villehardouin, le chroniqueur de l'époque, témoigne du fait que « depuis la création du monde, jamais on ne fit tant de butin dans une ville ».

3 L'accueil réservé à la toile fut mitigé. On critiqua les négligences du dessin, le manque de lisibilité de la composition, la couleur froide et terreuse, inhabituelle pour une scène orientale. Baudelaire, en revanche, lors de la présentation de la toile à l'Exposition universelle de 1855, donc quatorze ans plus tard, est enthousiasmé :

«Mais le tableau des Croisés est si profondément pénétrant, abstraction faite du sujet, par son harmonie orageuse et lugubre! Quel ciel et quelle mer! Tout y est tumultueux et tranquille, comme la suite d'un grand événement. La ville, échelonnée derrière les Croisés qui viennent de la traverser, s'allonge avec une prestigieuse vérité. Et toujours ces drapeaux miroitants, ondoyants, faisant se dérouler et claquer leurs polis lumineux dans l'atmosphère transparente! Toujours la foule agissante, inquiète, le tumulte des armes, la pompe des vêtements, la vérité 
emphatique du geste dans les grandes circonstances de la vie sont d'une beauté essentiellement shakespearienne! Car nul, après Shakespeare, n'excelle comme Delacroix à fondre dans une unité mystérieuse le drame et la rêverie $»^{1}$.

4 Par ailleurs, la technique très novatrice de Delacroix et l'interprétation qu'il donne du sujet apparaissaient aux yeux de la majorité de ses contemporains comme parfaitement incongrues au sein du musée historique de Versailles. L'opinion de Delacroix sur la peinture d'histoire ne peut aisément s'accorder avec celle de Louis-Philippe. Il ne s'agit pas pour le peintre d'illustrer de la manière la plus précise possible un événement, mais plutôt de mettre en valeur un moment exceptionnel de l'histoire, par les moyens expressifs propres à la peinture.

5 Sous le titre de Dialogue avec Eugène Delacroix sur l'entrée des croisés à Constantinople, l'écrivain contemporain Michel Butor se dégage du point de vue privilégié par l'historien ou par l'historien de l'art, à travers un savant collage de citations de tous genres et d'origines diverses qui constituent ce bref opuscule divisé en quatorze scènes, où le texte est entrecoupé de deux reproductions de l'immense toile réduite à la taille d'une carte postale, et de rappels de certains de ses détails isolés sur le blanc de la page, cette fois de la taille d'un timbre poste, ce qui va nous permettre de l'explorer et de la lire plus en détail.

Déjà le dialogue énoncé dans l'intitulé nous renvoie en premier lieu au dialogue socratique qui oppose l'entretien dialectique dont l'essence serait populaire et carnavalesque, au discours rhétorique, monologique, car, au lieu d'errer à travers le langage, la rhétorique étant l'art de la contrefaçon, l'entretien ou le dialogue mènent à la découverte de la vérité par une remise en question systématique de tout ce qui peut sembler définitivement établi. Le dialogue cultive l'ouverture à l'altérité qui le préserve de la fermeture sur soi, constituant ainsi le lien privilégié d'une rencontre entre le même et l'autre. «Son art est l'art de l'articulation du fantasme, de la corrélation des signes »². Mais le concept dialogique nous renvoie par ailleurs, à travers la pensée de Bakhtine, au dialogue intertextuel, ce processus par lequel un texte nouveau s'écrit à partir d'un autre, l'insère dans son espace et le modifie, se l'approprie, l'assimilant tout en le transformant : ce travail intertextuel engendre ces phénomènes que Kristeva nomme, selon Bakhtine, « dialogisme» et "polyphonie ». "L'observation doit porter, affirme-t-elle, sur le nouveau texte et sur les traces qu'il conserve d'éléments textuels antérieurs, sur l'interaction textuelle qui se produit à l'intérieur d'un texte ». Cependant Laurent Jenny dans «La stratégie de la forme» nuance et précise les affirmations de Kristeva en montrant les rapports qu'entretient l'intertextualité avec la critique des sources: "l'intertextualité désigne non pas une addition confuse et mystérieuse d'influences, mais le travail de transformation et d'assimilation de plusieurs textes opéré par un texte centreur qui garde le leadership du sens $»^{3}$.

7 Nous allons donc tenter de montrer à travers ce court texte de Michel Butor, comment le jeu de l'intertexte produit une théâtralisation du pictural qui mène à une conception renouvelée du carnavalesque.

8 Dès l'énonciation de ce que cherche à réaliser Michel Butor, la référence théâtrale se manifeste avec force :

" Je ne cherche pas ici à faire un travail d'historien de l'art, mais à dialoguer avec le

tableau, à l'animer devant vous. Ses figures sont comme les acteurs d'une troupe à

qui je vais distribuer les rôles qu'ils vont tenir devant ce magnifique décor $»^{4}$ 
La théâtralisation va, en effet, s'élaborer à deux niveaux, de façon complémentaire, celui de l'énoncé et celui de l'énonciation. L'énoncé se matérialise par ce que représente la peinture telle qu'interprétée à l'aide de l'ensemble des textes convoqués à l'élaboration de cette théâtralisation ; l'énonciation se traduirait par le traitement attribué à ces textes par la mise en scène butorienne. La théâtralisation des constituants référentiels qui résulte de la mise en scène des matériaux textuels impose donc une nouvelle vision des choses compatible avec ce que sous-tend une lecture moderne de la peinture de Delacroix, mais renvoyant de façon simultanée à ce que peut vouloir signifier la composition butorienne. Cette tension entre mimésis et textualité ne saurait cependant s'arrêter là : ainsi, le double référent, pictural et historique, se métamorphose en une nouvelle image, par le biais de tout un travail de retextualisation de la matière mémorielle. La scène renouvelée issue de ce curieux dialogue, puisqu'il se rapproche étrangement d'un polylogue très ouvert, polymorphe, auquel nous sommes conviés, relève à la fois de l'historique, du pictural, du scriptural et même de l'autobiographique pour se condenser dans une dimension à caractère proprement mythique.

10 Après avoir clarifié la confusion généalogique qui peut se dégager, pour le profane, de la dynastie des Comnène et rappelé par la bouche de Voltaire les événements essentiels de la prise de Constantinople par les croisés, le 13 avril 1204, donc neuf mois après leur entrée dans la ville, le 18 juillet 1203, Butor, montrant que ces deux événements se super posent dans le souvenir historique et dans l'imagination de Delacroix, nous invite, par le biais du tableau, à une reconstitution de la scène à la fois au sens historique et au sens théâtral du mot, en privilégiant la première entrée, moins barbare et moins sanguinaire que la seconde.

11 Partant des quatre chevaux situés au centre du tableau, il procède à une première étape de la théatralisation de la peinture, attribuant à chacune des figures représentées un rôle propre à l'histoire de la IV croisade, et identifiant drapeaux et oriflammes «qui font claquer leurs plis lumineux dans l'atmosphère transparente ».

12 La constatation que tous ces acteurs de haut lignage sont, «à cette époque », usurpateurs et assassins, fait en sorte que la description des événements historiques se donne à lire comme s'il s'agissait d'un drame shakespearien. Les personnages que l'on ne saurait qualifier de secondaires, mais qui ne font pas partie du groupe central de cavaliers, de chefs militaires, se voient, eux aussi, attribuer des rôles : ainsi, le vieillard au somptueux costume, agenouillé, s'appuyant sur une femme, et dont le geste de la main peut suggérer qu'il est aveugle, devient le vieil empereur déchu, Isaac Ange Comnène et rapproché d'Oedipe à Colonne appuyé sur son Antigone implorant un nouveau Thésée, alors que les historiens de l'art rapprochaient, plutôt, ce personnage de Belisaire, le vieillard aveugle que David avait dépeint dans une toile du Salon de 1781, y voyant une allusion qui renforce le caractère dénonciateur de l'œuvre de Delacroix.

13 Le patriarche à la gauche de la toile, l'usurpateur Murzuphle, les impératrices et même le guerrier et chroniqueur Robert de Clari sont, eux aussi, chacun, identifiés.

14 Le décor où se déroule la scène est ensuite élaboré : une première étape pose le portique à colonnes de saphyre que l'on voit au premier plan, à gauche dans le tableau de Delacroix, et le place dans la filiation des œuvres de Claude Lorrain, alors que la source stylistique majeure à laquelle Delacroix se réfère, selon les spécialistes, serait plutôt la grande toile de Véronèse du Louvre, Les noces de Cana. 
distanciation à la manière brechtienne, en décrivant divers aspects du traitement que
l'auteur fait subir aux textes qu'il utilise. «Il va de soi que j'adopte le texte ancien » écrit Butor (p. 46) à propos de Villehardouin et (p. 71) «Comme pour Villehadouin j'adapte toujours son texte pour pouvoir le citer » à propos, cette fois, du témoignage de Robert de Clari, comme si l'adaptation était préalable à toute citation. Une étude des manipulations que subit l'intertexte montre que la reprise de chacune des citations s'effectue selon une certaine méthode qui implique techniques et procédés spécifiques: celles de Charles Fourier sont précédées de commentaires qui en clarifient le sens (p. 12 et 13); celles de Voltaire sont contextualisées (p.17); les inexactitudes perçues à travers celles de Huysmans sont corrigées (p.44); le texte de Villehardouin se trouve parfois résumé, parfois cité, mais l'ordre des paragraphes en a été modifié, ou une partie du texte d'origine a été omise entre deux paragraphes cités ; ailleurs, par un procédé d'intercalage, la voix de Voltaire ou celle de de Clari interrompent le célèbre chroniqueur et prennent la relève.

18 Ainsi, la mise en scène qu'imposent le découpage et la manipulation méthodique imprimée aux textes cités relève du travail de composition propre à celui du peintre ou mieux encore du collagiste. Le texte butorien, dont la surface est contaminée par cet effet de prélèvement, se donne donc à lire comme l'articulation de ces voix au timbre distinct, comme véritable polyphonie. Mais un autre effet que provoque cette contamination avouée, est de dénoter la distance que prend l'écrivain à l'égard des textes qu'il utilise pour architecturer celui qu'il nous donne à lire.

19 Nous commençons à découvrir que ce court récit se présente comme une juxtaposition collective de récits dont les citations s'exhibent comme telles, un ensemble dialogique dont semblent se dégager, trois constellations, l'une gravitant autour de la peinture de Delacroix, l'autre fouillant l'histoire événementielle de l'entrée et de la prise de Constantinople, la dernière explorant la ville même de Constantinople. Le premier ensemble suit le processus de l'œuvre, depuis l'argument qui a été proposé à Delacroix et qui sert de description dans le livre du Salon de 1841 où le tableau est exposé (p. 9), en passant par la brève mention qu'en fait l'artiste dans sa correspondance (p.188), sans oublier le double titre qui la désigne, celui sous lequel elle a été exposée en 1841 « Prise de Constantinople par les croisés » et celui qui a, peu à peu, prévalu, que l'on retrouve aujourd'hui sur le cadre, selon Butor, "L'entrée des croisés à Constantinople». À ces textes directement rattachés à la peinture, s'ajoutent ceux qui ont été inspirés par l'œuvre, par ce qu'elle représente, mais aussi par sa manière de représenter : « Le compte rendu de l'exposition universelle de 1855 » (p. 35 à 37) qu'écrivit Baudelaire enthousiaste, la description que l'on retrouve dans Certains, une œuvre de Huysmans (p. 38 à 52), ainsi 
que le témoignage de Cézanne, dont les propos sont rapportés par Joachim Gasquet, au sujet de l'extinction des couleurs que le vieillissement a causée à cette œuvre (p. 101).

Un second regroupement de textes va se déployer, non plus à partir de la peinture, mais bien, cette fois, de ce qui y est représenté. Deux textes critiques, voire hostiles aux croisades sont convoqués, l'un (p.13) provenant du Nouveau monde amoureux de Charles Fourier, que le peintre n'a évidemment pu lire, l'autre de Voltaire, extrait de l'Essai sur les mours, qu'il aurait pu consulter. Par ailleurs, Butor utilise deux chroniques célèbres qui constituent des témoignages, l'une est le récit de Geoffroy de Villehardouin qui joua un rôle important comme chef militaire et, plus encore, comme diplomate dans la IV ${ }^{\mathrm{e}}$ Croisade ; l'autre est le récit du guerrier Robert de Clari qui, plutôt que de voir les choses de haut sans se perdre dans les détails, témoigne des croisés de seconde catégorie, ceux entre la piétaille et les grands, au sujet desquels il nous renseigne.

Un troisième groupe de textes porte sur la ville de Constantinople. Évidemment, tous les récits de la prise de cette ville la décrivent d'une manière ou d'une autre, mais certains passages du texte de Robert de Clari s'émerveillent devant ses richesses dont il nous décrit le luxe inouï (p. 72 à 76). Butor fait aussi intervenir d'autres descriptions qui, de prime abord, peuvent sembler anodines : ainsi, ce guide d'Istambul par Ernest Mamboury (p. 56 à 60), édition mise à jour en fin mars 1951, précise-t-il. Or ce texte permet une incursion dans un autre ensemble d'écrits qui ne sont pas évoqués de façon directe, bien que le contexte y renvoie de manière oblique, puisqu'il s'agit des textes que Butor a écrits sur Constantinople ou Istambul où il a séjourné vers 1955, à commencer par le premier tome du Génie du lieu (1958), et par Description de San Marco (1963) :

"Ce sont trois villes qui se superposent et que l'on démêle en errant, trois villes de structure profondément différentes, trois villes nées de trois invasions. Insistons encore sur la dernière, poursuit-il, l'industrielle, la bancaire, la noire [...] $»^{5}$.

ce qui l'amène à décrire Istambul comme un Liverpool d'Orient. Par ailleurs, sa lecture de la célèbre Basilique nous décrit la pièce maîtresse de la façade, les quatre chevaux de bronze au dessus du portail principal (p. 14) et qui y ont été apportés par le doge Enrico Dandolo à son retour de Constantinople :

«Pièce maîtresse de la collection: les quatre chevaux de bronze au-dessus du portail principal, le seul quadrige antique subsistant, œuvre grecque, pense-t-on, du $\mathrm{IV}^{\mathrm{e}}$ ou $\mathrm{III}^{\mathrm{e}}$ siècle avant Jésus-Christ, pièce disputée au long des âges, déjà repérée sans doute par Néron pour couronner son arc de triomphe, transportée par Constantin dans sa nouvelle Rome où elle couronnait l'hippodrome, et raflée en dernier lieu par Napoléon pour l'arc de triomphe du Carrousel où elle resta jusqu'à ce que le congrès de Vienne en eût ordonné la restitution $»^{6}$.

C'est à ce moment que le jeune Delacroix a pu les admirer lorsqu'il avait entre dix et dixsept ans. Ailleurs ce sont les colonnes

«dont les chapiteaux supportent quatre colonnes, elles-mêmes à chapiteaux, tout cela pièce de collection, ramenées de Constantinople en 1204 lors de cette expédition de pillage en quoi s'était transformée la quatrième croisade sous la direction du doge Enrico Dandolo $»^{7}$.

On le voit, autant de motifs qui architecturent la toile de Delacroix.

Plus qu'une condensation des évènements et des personnages, la peinture illustre aussi une condensation du temps : les quatre incendies que Butor y dénombre ont bien eu lieu, mais en quatre temps différents si l'on en croit les chroniques du temps. La mise en scène de l'Histoire par la peinture relève donc d'un processus de déréalisation du référent. Ainsi, Théophile Gauthier qui, lui, est allé à Istambul contrairement à Delacroix, s'étonne 
du traitement qu'il a pu donner au ciel de cette ville que Butor rapproche du ciel parisien, voyant dans cette peinture une allusion très nette à la révolution de 1830 avec son agitation, ses drapeaux rouges et noirs, alors qu'ils sont véritablement bleu foncé, ses incendies. La mise en scène butorienne s'efforce de démonter le jeu d'illusions référentielles qui nous obscurcit la vue, et sous lequel Delacroix actualise le sujet représenté, le rendant anecdotique, bien qu'il reste toujours investi d'une forte valeur symbolique. Rien ne nous interdit de croire que le texte de Butor ne joue pas le même rôle à notre époque : l'analogie théâtrale n'est pas sans poser un discours sur l'Histoire et cette remembrance d'un moment capital de l'histoire occidentale ne nous convierait-elle pas à un certain questionnement? Car derrière l'esquisse de ces figures du drame Shakespearien, c'est un véritable théâtre de la cruauté qui se met en branle. D'un côté nous avons la troupe des Croisés envahisseurs, avides calculateurs, voleurs, violeurs et assassins ; à l'opposé, du côté de la dynastie des Comnènes à Constantinople, jugez-en par vous-même: Nous avons Alexis II, tué par son oncle Andronic Comnène ; cet usurpateur sera tué après avoir été aveuglé par Isaac l'Ange, qui sera lui-même aveuglé par son frère Alexis III l'Ange Comnène. Le fils d'Isaac l'Ange, le jeune Alexis III, sera étranglé par l'un de ses parents, Alexis IV Dukas Comnène, surnommé Murzuphle; cet usurpateur sera à son tour fait prisonnier et aveuglé par Alexis III l'Ange Comnène. Il mourra en prison avec sa femme où les aura envoyés Boniface de Montferrat. À cette évocation, on lit différemment la huitième strophe des Phares de Baudelaire, le mot " ange » y prenant un sens bien spécifique et son commentaire dans le compte rendu de l'Exposition universelle de 1855 :

«Delacroix, lac de sang hanté de mauvais anges,

Ombragé par un bois de sapin toujours vert

Où, sous un ciel chagrin, des fanfares étranges

Passent, comme un soupir étouffé de Weber $\rrbracket^{8}$.

«Lac de sang: le rouge; - hanté de mauvais anges: surnaturalisme; - un bois toujours vert: le vert, complémentaire du rouge: - un ciel chagrin: les fonds orageux et tumultueux de ses tableaux; - les fanfares de Weber : idées de musique romantique que réveillent les harmonies de sa couleur $"$.

Or, ce qui semble frapper Butor, c'est la répétition de l'histoire, son apparent mouvement cyclique, son éternel retour, qui se manifeste derrière la métaphore bien réelle de l'aveuglement :

«Que d'yeux crevés dans toute cette histoire ! La splendeur picturale se déploie sur un fond d'aveuglement: yeux crevés du frère d'Isaac, yeux arrachés d'Andronic, yeux crevés de Murzufle, et naturellement les yeux crevés de notre empereur agenouillé, désespéré, Isaac Ange qui recommence l'histoire d'Alexis II qui avait fait revenir auprès de lui celui qui allait être son pire ennemi Andronic, qui préfigure l'histoire de son propre fils Alexis IV qui ferait revenir auprès de lui celui qui allait être son pire ennemi Alexis V Dukas Murzufle.[.... $]^{10}$.

Déjà, commentant Geoffroy de Villehardouin, il nous avait brièvement expliqué la technique utilisée : « (en réalité les Byzantins de l'époque avaient l'habitude de fendre les yeux avec un fer rouge, ou de les arroser de vinaigre bouillant)». Plus loin, lorsqu'il évoque le supplice d'Andronic, il cite Voltaire : «On traîna l'empereur Andronic dans les rues, on lui coupa une main, on lui creva les yeux, on lui versa de l'eau bouillante sur le corps, et il expira dans les plus cruels supplices $»^{11}$. et résume en ses mots le récit de Robert de Clari qui le dépasse largement dans l'horreur : « la foule, nous dit-il, le met en pièces à tel point qu'il ne reste plus que ses os qui sont jetés dans une latrine. Cette fois les yeux ont pu être littéralement arrachés $»^{12}$. 
28 Et renvoie, sans les citer, aux dernières pages de Salammbô. Or, curieusement, ces dernières pages du chef-d'œuvre de Flaubert, plutôt que d'illustrer un paroxystique aveuglement à travers le récit atroce du supplice de Mâtho, nous en offrent plutôt le négatif :

Il n'avait plus, sauf les yeux, d'apparence humaine; c'était une longue forme complètement rouge; ses liens rompus pendaient le long de ses cuisses, mais on ne les distinguait pas des tendons de ses poignets tout dénudés; sa bouche restait grande ouverte; de ses orbites sortaient deux flammes qui avaient l'air de monter jusqu'à ses cheveux ; - et le misérable marchait toujours !13

Comme si sous cette fête de la cruauté s'énonçait une question essentielle : comment se dégager d'une conception de l'Histoire comme lieu de répétition et d'enfermement ? La théâtralisation qui associe déréalisation du référent et mise en scène de la répétition de l'Histoire ne peut donc qu'avoir recours à la mythologie : le mythe n'entraîne-t-il pas la sortie hors de l'Histoire?

L'élaboration du mythe va donc se construire à partir des motifs suivants :

31 Nous avons déjà l'écorché vif qui oppose son regard de feu à l'aveuglement général, mais qui, dans un autre registre, fait la jonction avec le carnavalesque que nous évoquions au début: n'oublions pas que le mot «carnaval » vient de l'italien carnevale que le gênois a conservé, altération de carneleva qui signifie « enlève-chair ».

Nous avons ensuite la signature de Delacroix. Dans Les mots dans la peinture, sous le titre de «la signature entre deux mondes» Butor analysait la signature de Delacroix dans Le 28 juillet 1830 - La liberté guidant le peuple dont

«le relèvement [...], rouge sang, est d'autant plus sensible qu'elle apparaît juste derrière l'horizontale d'une barricade; la date « 1830 » est bien peinte comme un soulèvement.

Mais si elle peut apparaître «derrière » c'est qu'elle s'inscrit [...] sur un objet qui doit se situer dans l'espace représenté ${ }^{14}$.

33 Ce qui attire le regard de Butor dans L'Entrée des Croisés à Constantinople est moins la signature de Delacroix que son redoublement hiéroglyphique inscrit sur le costume du patriarche indigné à gauche de la scène :

« Remarquons qu'au milieu de tous ces croisés, il est le seul à être orné de la croix, la croix grecque à quatre barres égales $»^{15}$.

Or, celui qui participe au carnaval n'est-il pas à la fois acteur et spectateur?

«Il perd sa conscience de personne pour passer par le zéro de l'activité carnavalesque et se dédoubler en sujet du spectacle et objet du jeu. Dans le carnaval le sujet est anéanti : là s'accomplit la structure de l'auteur comme anonymat qui crée et se voit créer, comme moi et comme autre, comme homme et comme masque $»^{16}$.

35 Enfin, nous avons le personnage de l'enfant, à la droite du tableau, dont le rôle n'est déterminé que dans la dernière scène du texte de Butor, intitulée "L'espoir de Byzance ", associant le téléologique à l'archéologique puisque le dialogue y est élaboré précisément " dans l'intention de ", mais avec un retour aux origines à une époque d'avant le schisme :

« Regardez-le délicieusement esquissé, tranquille dans les bras de sa mère, à peu près à mi-hauteur, au bord à droite, entre incendiaire et incendies, impératrices et faubourgs, à l'écart de cette foule d'empereurs qui tombent les uns sur les autres comme les cartes d'un château. J'y reconnais ma préhistoire, nous dit Butor. C'est le rôle que j'adopte dans cette troupe, et c'est sous ce masque en bourgeon que je vous invite à vous installer $\aleph^{17}$. 
Ainsi, celui ou celle qui regarde le tableau, le décrypte, le décrit, se voit transformé en l'un de ses personnages, acteur en devenir, régénéré par le regard de feu de l'écorché vif, convié à participer à ce jeu de rôles, à s'intégrer à la scène, à offrir une alternative à ces personnages théâtralisés en amorçant une mise en mouvement de cet ensemble de figures exemplaires, modèle réduit de l'humanité, tristes tragédiens condamnés sinon à réitérer l'Histoire.

\section{NOTES}

1. Charles Baudelaire, «Exposition universelle, 1855, Beaux-Arts », III Eugène Delacroix in Euvres complètes, Paris, 1980, Robert Laffont, coll. Bouquins p. 733.

2. Julia Kristeva, « Le mot, le dialogue et le roman », in Séméiotikè, Paris, Éd. du Seuil, coll. Points n 96, 1969, p. 102.

3. Laurent Jenny, « La stratégie de la forme » in Poétique n 27, Paris, Éd. du Seuil, 1976,

p. 257.

4. Michel Butor, Dialogue avec Eugène Delacroix sur l'entrée des croisés à Constantinople, SaintEtienne, S'Printer L'Édition, 1991, p. 10.

5. Michel Butor, « Istambul », in Le génie du lieu, Paris, 1958, Grasset, coll. La Galerie, p. 33.

6. Michel Butor, Description de San Marco, Paris, 1963, Gallimard, p. 14-15.

7. Michel Butor, Description de San Marco, op. cit., p. 15.

8. Charles Baudelaire, « Les phares », in Euvres complètes, op. cit., p. 10.

9. Charles Baudelaire, « Exposition... » in Cuvres complètes, op. cit., p. 734.

10. Michel Butor, Dialogue..., op. cit., p. 94, 95.

11. Michel Butor, op. cit., p. 93.

12. Michel Butor, op. cit., p. 93.

13. Gustave Flaubert, Salammbô, Paris, 1964, Garnier Flammarion, p. 310.

14. Michel Butor, Les mots dans la peinture, Genève, 1969, Albert Skira éditeur, p. 114.

15. Michel Butor, Dialogue... op. cit., p. 53.

16. Julia Kristeva, op. cit., p. 99.

17. Michel Butor, Dialogue..., op. cit., p. 120.

\section{RÉSUMÉS}

Sous couvert d'un dialogue avec Eugène Delacroix, l'auteur Michel Butor, dans un opuscule, se propose d'animer une œuvre picturale reliée à un événement historique d'importance, l'Entrée des Croisés à Constantinople. Le court texte divisé en 14 scènes propose moins un travail d'historien de l'art qu'un dialogue avec le tableau dont les « figures sont comme les acteurs d'une troupe à 
qui (il va) distribuer les rôles qu'ils vont tenir dans ce magnifique décor ». Pour réaliser cette mise en scène de l'Histoire qui devient lecture régénératrice, il convoque un ensemble de textes évoquant chacun une vision du monde. Ce polylogue composé de citations, paraphrases, résumés, commentaires utilisés comme autant de répliques, décrit à la fois un événement historique et une toile célèbre en mouvement. Mais au-delà du descriptif, le jeu intertextuel produit une théâtralisation du pictural et mène à une conception renouvelée de la carnavalisation.

\section{AUTEUR}

JACQUES LA MOTHE

Université du Québec à Montréal 